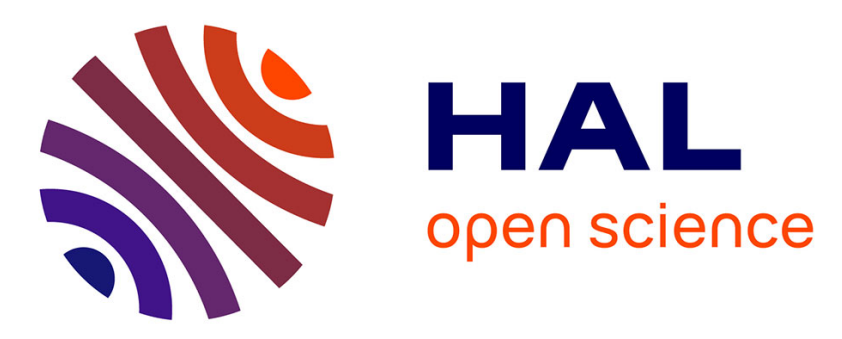

\title{
An Upper Bound on Antenna Near-Field Deviations Caused by Mutual Coupling for Applications in Optimization Design Methods
}

François Sarrazin, Said Mikki, P Pouliguen, A Sharaiha, Yahia Antar

\section{- To cite this version:}

François Sarrazin, Said Mikki, P Pouliguen, A Sharaiha, Yahia Antar. An Upper Bound on Antenna Near-Field Deviations Caused by Mutual Coupling for Applications in Optimization Design Methods. IEEE Antennas and Wireless Propagation Letters, 2016, 15, pp.429-432. 10.1109/LAWP.2015.2450692 . hal-01435111

\section{HAL Id: hal-01435111 \\ https://hal.science/hal-01435111}

Submitted on 13 Jan 2017

HAL is a multi-disciplinary open access archive for the deposit and dissemination of scientific research documents, whether they are published or not. The documents may come from teaching and research institutions in France or abroad, or from public or private research centers.
L'archive ouverte pluridisciplinaire HAL, est destinée au dépôt et à la diffusion de documents scientifiques de niveau recherche, publiés ou non, émanant des établissements d'enseignement et de recherche français ou étrangers, des laboratoires publics ou privés. 


\title{
An Upper Bound on Antenna Near-Field Deviations Caused by Mutual Coupling for Applications in Optimization Design Methods
}

\author{
Francois Sarrazin, Said Mikki, Philippe Pouliguen, Ala Sharaiha, Senior Member, IEEE, and Yahia \\ Antar, Life Fellow, IEEE
}

\begin{abstract}
The present letter studies the impact of electromagnetic mutual coupling on the near fields of generic antenna systems. The main objective here is to derive an upper bound on near-fields deviations caused by mutual coupling. The method transforms the problem of near-field coupling from the near zone to the recently introduced antenna current Green's function defined on the antenna itself. Through this upper bound, the formulation presented here can be used to develop automatic algorithms for the design of coupled antenna systems with optimum near-field performance. Numerical examples involving linear wire arrays are given to demonstrate and confirm the approach proposed here.
\end{abstract}

Index Terms-Antenna current Green's function, mutual coupling, near field.

\section{INTRODUCTION}

$\mathrm{M}$ UTUAL coupling in electromagnetic systems is one of the most important and fundamental problems involved in the design and development of systems for diverse applications ranging from radar and sensor devices to wireless communications such as multiple-input-multiple-output (MIMO) and mobile links. The ongoing demand to reduce the size of these systems, together with the need to explore new functions and potentials in crowded electromagnetic environments and subwavelength behavior, have made necessary undertaking a reconsideration of the near field associated with interacting antennas [1]-[7]. Although mutual coupling is traditionally characterized by circuit parameters, i.e., mutual impedances [8]-[10] or the embedded radiation pattern method [11], it has been pointed out recently that deviations in device performance in the near zone due to coupling effects should be treated by more comprehensive

This work was financially supported in part by the Direction Generale de l'Armement (DGA), France.

F. Sarrazin was with the Institute of Electronics and Telecommunications of Rennes, University of Rennes 1, France. He is now with the Royal Military College, Kingston, Canada (e-mail: francois.sarrazin@rmc.ca).

S. Mikki and Y. Antar are with the Royal Military College, Kingston, Canada (e-mail: said.mikki@rmc.ca; antar-y@rmc.ca).

P. Pouliguen is with the Direction Generale de l'Armement, Bagneux, France (e-mail: philippe.pouliguen@intradef.gouv.fr).

A. Sharaiha is with the Institute of Electronics and Telecommunications of Rennes, University of Rennes 1, France (e-mail: ala.sharaiha@univrennes1.fr). methods [1], [2], [6]. In particular, new emerging applications like near-field communication (NFC), near-field MIMO, the physics of complex media, and wireless power transfer have all already suggested the need to reexamine the problem of computing and characterize near-field effects caused by mutual coupling and energy exchange at short distances.

Though near fields are routinely computed in full-wave analysis codes such as finite element method (FEM), method of moments (MoM), and finite-difference time domain (FDTD), to our knowledge, rigorous and comprehensive studies of the impact of mutual coupling on near fields has been taken up systematically only recently. Specifically, the work in [6] proposed a method to estimate mutual coupling in the near zone using a single numerical measure [global error in the observation region, see (6) below]. On the other hand, using the method of the antenna current Green's function (ACGF) [2], the new approach of characterizing mutual coupling in terms of a special Green's function, the mutual coupling Green's function (basically, an exact spatial transfer function for mutual coupling effects on the antenna current) was proposed and verified in [3].

In the present letters, the proposals of modeling mutual coupling through a special ACGF [3] and characterizing mutual coupling in the near-field zone [6] will be combined here to analyze and understand the near field of coupled antenna arrays at a very general level. Indeed, we show that the deviation of the near field from its ideal value due to mutual coupling effects computed using the method in [6] is bounded by a quantity that depends on the mutual coupling ACGF defined on the entire antenna surface [3]. This upper bound can be used to provide a quick characterization of near fields' sensitivity to electromagnetic mutual coupling effects and to suggest novel methods for properly designing the radiating elements in antenna arrays in order to meet given performance measures determined in terms of the near field, for example near-field focusing and communications. Furthermore, this letter provides a theoretical frame- work for developing special design methods, for example engineering the current on the antenna, to obtain antennas with optimum near-field coupling performance. The present letter provides proof of concept and simple example for demonstration, while detailed investigations of more throughout methods to compensate for near-field mutual coupling will be given in future work. 


\section{Derivation Of The Upper Bound Using The Antenna CURRENT GREEN'S FUNCTION METHOD}

The key idea in this letter is to use the ACGF instead of the current or the impedance parameters of the antenna in order to study the mechanism behind near-field deviations in electromagnetically coupled antennas. The motivation is that in order to be able to derive an upper bound on the near-field error valid for arbitrary-shaped excitation, one must incorporate in the derivation itself variations in the input signal exciting the antenna itself. Therefore, one needs the transfer function that connects this input excitation with the actual current induced on the antenna (the latter is responsible of producing the near field.) This special transfer function is in fact the ACGF of the problem [2]. The input impedance method includes only the level of the excitation in the derivation; therefore, it cannot deal with arbitrary forms of excitation (i.e., both level and shape of excitation are needed to insure the validity of the near-field study for any input excitation or illuminating scattered fields by objects close to the antenna under consideration.)

The current $\mathbf{J}(\mathbf{r})$ induced on the antenna due to excitation field $\mathbf{E}^{\text {ex }}(\mathbf{r})$ applied at a port area $U$ is given by [2]-[3]

$$
\mathbf{J}(\mathbf{r})=\int_{U} \overline{\mathbf{F}}\left(\mathbf{r}, \mathbf{r}^{\prime}\right) \cdot \mathbf{E}^{\mathrm{ex}}\left(\mathbf{r}^{\prime}\right) d s^{\prime},
$$

where the tensor (dyad) $\overline{\mathbf{F}}\left(\mathbf{r}, \mathbf{r}^{\prime}\right)$ is the ACGF of the system under consideration. Throughout this paper, a time harmonic excitation $\exp (-i \omega t)$ is assumed and suppressed everywhere. In particular, note that the ACGF is frequency dependent.

Consider two arbitrary antennas A and B brought into proximity to each other in order to allow for mutual coupling effects to come into being. For simplicity, we assume that only antenna $\mathrm{A}$ is excited and denotes this excitation by $\mathbf{E}^{\mathrm{ex}}(\mathbf{r})$ as above. The ACGF of antenna A without mutual coupling is denoted by $\overline{\mathbf{F}}_{0}^{A}\left(\mathbf{r}, \mathbf{r}^{\prime}\right)$. However, due to mutual coupling, the system $\mathrm{A}+\mathrm{B}$ is now described by the new ACGF

$$
\overline{\mathbf{F}}^{\prime}\left(\mathbf{r}, \mathbf{r}^{\prime}\right)=\overline{\mathbf{F}}^{A}\left(\mathbf{r}, \mathbf{r}^{\prime}\right)+\overline{\mathbf{F}}^{B A}\left(\mathbf{r}, \mathbf{r}^{\prime}\right)
$$

Following [3], the mutual coupling ACGF is defined as

$$
\delta \overline{\mathbf{F}}\left(\mathbf{r}, \mathbf{r}^{\prime}\right):=\overline{\mathbf{F}}^{\prime}\left(\mathbf{r}, \mathbf{r}^{\prime}\right)-\overline{\mathbf{F}}_{0}^{A}\left(\mathbf{r}, \mathbf{r}^{\prime}\right)=\delta \overline{\mathbf{F}}^{A}\left(\mathbf{r}, \mathbf{r}^{\prime}\right)+\overline{\mathbf{F}}^{B A}\left(\mathbf{r}, \mathbf{r}^{\prime}\right) .
$$

In other words, $\delta \overline{\mathbf{F}}\left(\mathbf{r}, \mathbf{r}^{\prime}\right)$ is the difference in value between the ACGFs before and after mutual coupling. Note that $\delta \overline{\mathbf{F}}^{A}\left(\mathbf{r}, \mathbf{r}^{\prime}\right):=\overline{\mathbf{F}}^{A}\left(\mathbf{r}, \mathbf{r}^{\prime}\right)-\overline{\mathbf{F}}_{0}^{A}\left(\mathbf{r}, \mathbf{r}^{\prime}\right) . \quad$ Moreover, $\quad \overline{\mathbf{F}}^{B A}\left(\mathbf{r}, \mathbf{r}^{\prime}\right)$ physically means the transfer function connecting an excitation on antenna $A$ to the response current induced on antenna B. A general method to compute $\delta \overline{\mathbf{F}}\left(\mathbf{r}, \mathbf{r}^{\prime}\right)$ using perturbation theory was proposed in [3].

The field radiated by an antenna is given by [1], [5]

$$
\mathbf{E}(\mathbf{r})=\int_{S} \overline{\mathbf{G}}\left(\mathbf{r}, \mathbf{r}^{\prime}\right) \cdot \mathbf{J}\left(\mathbf{r}^{\prime}\right) d s^{\prime},
$$

where $\overline{\mathbf{G}}\left(\mathbf{r}, \mathbf{r}^{\prime}\right)$ is the free-space dyadic Green's function [1] and $S$ is the radiating antenna's full surface. Because of mutual coupling, the current induced on the antenna system changes as $\mathbf{J}(\mathbf{r}) \rightarrow \mathbf{J}^{\prime}(\mathbf{r})$; hence we define the deviation in the current due to mutual coupling as $\delta \mathbf{J}(\mathbf{r}):=\mathbf{J}^{\prime}(\mathbf{r})-\mathbf{J}(\mathbf{r})$. Similarly, mutual coupling changes the field according to the scheme $\mathbf{E}(\mathbf{r}) \rightarrow \mathbf{E}^{\prime}(\mathbf{r})$ and we define $\delta \mathbf{E}(\mathbf{r}):=\mathbf{E}^{\prime}(\mathbf{r})-\mathbf{E}(\mathbf{r})$. From (1) and (4), we therefore have

$$
\begin{aligned}
& \delta \mathbf{J}(\mathbf{r})=\int_{U} \delta \overline{\mathbf{F}}\left(\mathbf{r}, \mathbf{r}^{\prime}\right) \cdot \mathbf{E}^{\mathrm{ex}}\left(\mathbf{r}^{\prime}\right) d s^{\prime}, \\
& \delta \mathbf{E}(\mathbf{r})=\int_{S} \overline{\mathbf{G}}\left(\mathbf{r}, \mathbf{r}^{\prime}\right) \cdot \delta \mathbf{J}\left(\mathbf{r}^{\prime}\right) d s^{\prime}
\end{aligned}
$$

Note the formal similarity between (5) and (6), which was one of the motivations for introducing the ACGF formalism in [2].

For a generic input excitation, we assume that the energy of the source is finite, i.e., we have

$$
\left\|\mathbf{E}^{\mathrm{ex}}\left(\mathbf{r}^{\prime}\right)\right\| \leq C,
$$

Here, $\|\cdot\|$ stands for the Euclidean vector norm and $C$ is a positive real number characterizing the maximum energy level of the input. From (6), we can write

$$
\|\delta \mathbf{E}(\mathbf{r})\| \leq \int_{S}\left\|\overline{\mathbf{G}}\left(\mathbf{r}, \mathbf{r}^{\prime}\right) \cdot \delta \mathbf{J}\left(\mathbf{r}^{\prime}\right)\right\| d s^{\prime}
$$

In order to proceed further, we need to evaluate the norm of a dyadic function multiplied by a vector. This can be accomplished in the following manner. First expand the dyadic multiplication as [1]

$$
\overline{\mathbf{G}}\left(\mathbf{r}, \mathbf{r}^{\prime}\right) \cdot \delta \mathbf{J}\left(\mathbf{r}^{\prime}\right)=\sum_{n, m} \mathbf{G}_{n}^{1}\left[\mathbf{G}_{m}^{2} \cdot \delta \mathbf{J}\left(\mathbf{r}^{\prime}\right)\right],
$$

where we used $\overline{\mathbf{G}}\left(\mathbf{r}, \mathbf{r}^{\prime}\right)=\sum_{n, m} \mathbf{G}_{n}^{1}\left(\mathbf{r}, \mathbf{r}^{\prime}\right) \mathbf{G}_{m}^{2}\left(\mathbf{r}, \mathbf{r}^{\prime}\right)$, and each of $n$ and $m$ ranges over the three Cartesian components. Therefore, from the usual properties of the norm, we find

$$
\left\|\overline{\mathbf{G}}\left(\mathbf{r}, \mathbf{r}^{\prime}\right) \cdot \delta \mathbf{J}\left(\mathbf{r}^{\prime}\right)\right\| \leq\left\|\delta \mathbf{J}\left(\mathbf{r}^{\prime}\right)\right\| \sum_{n, m}\left\|\mathbf{G}_{n}^{1}\right\|\left\|\mathbf{G}_{m}^{2}\right\|
$$

Next, we observe that the $\mathbf{r}$-function $\overline{\mathbf{G}}\left(\mathbf{r}, \mathbf{r}^{\prime}\right)$ is continuous in any compact subset of the exterior region, i.e., in any $D \subset V^{\text {exter }}$. The same is true for $\mathbf{r}^{\prime} \in S$ and also in the joint region $D \times S$. Therefore, $\overline{\mathbf{G}}\left(\mathbf{r}, \mathbf{r}^{\prime}\right)$ attains its maximum there. Denote this maximum by $M$, i.e., we may write 


$$
M:=\max _{\left(\mathbf{r}, \mathbf{r}^{\prime}\right) \in D \times S} \sum_{n, m}\left\|\mathbf{G}_{n}^{1}\left(\mathbf{r}, \mathbf{r}^{\prime}\right)\right\|\left\|\mathbf{G}_{m}^{2}\left(\mathbf{r}, \mathbf{r}^{\prime}\right)\right\| .
$$

From (8), (10), and (11), we conclude

$$
\|\delta \mathbf{E}(\mathbf{r})\| \leq M \int_{S}\left\|\delta \mathbf{J}\left(\mathbf{r}^{\prime}\right)\right\| d s^{\prime} .
$$

We now turn to (5), which allows us to write

$$
\|\delta \mathbf{J}(\mathbf{r})\| \leq \int_{U}\left\|\delta \overline{\mathbf{F}}\left(\mathbf{r}, \mathbf{r}^{\prime}\right) \cdot \mathbf{E}^{\mathrm{ex}}\left(\mathbf{r}^{\prime}\right)\right\| d s^{\prime}
$$

It was shown in [2] that the ACGF can always be put in a 3D dyadic form. Therefore, by treating the dyadic function $\delta \overline{\mathbf{F}}\left(\mathbf{r}, \mathbf{r}^{\prime}\right)$ in exactly the same way we dealt with $\overline{\mathbf{G}}\left(\mathbf{r}, \mathbf{r}^{\prime}\right)$ in (9), we find

$$
\left\|\delta \overline{\mathbf{F}}\left(\mathbf{r}, \mathbf{r}^{\prime}\right) \cdot \mathbf{E}^{\mathrm{ex}}\left(\mathbf{r}^{\prime}\right)\right\| \leq\left[\sum_{n, m}\left\|\delta \mathbf{F}_{n}^{1}\right\|\left\|\delta \mathbf{F}_{m}^{2}\right\|\right]\left\|\mathbf{E}^{\mathrm{ex}}\left(\mathbf{r}^{\prime}\right)\right\|,
$$

where the expansion $\delta \overline{\mathbf{F}}\left(\mathbf{r}, \mathbf{r}^{\prime}\right)=\sum_{n, m} \delta \mathbf{F}_{n}^{1}\left(\mathbf{r}, \mathbf{r}^{\prime}\right) \delta \mathbf{F}_{m}^{2}\left(\mathbf{r}, \mathbf{r}^{\prime}\right)$ from [2] was employed. Using (7) and substituting back to (13), we find

$$
\|\delta \mathbf{J}(\mathbf{r})\| \leq C \int_{U} \sum_{n, m}\left\|\delta \mathbf{F}_{n}^{1}\left(\mathbf{r}, \mathbf{r}^{\prime}\right)\right\|\left\|\delta \mathbf{F}_{m}^{2}\left(\mathbf{r}, \mathbf{r}^{\prime}\right)\right\| d s^{\prime} .
$$

Finally, (12) and (15) together yield

$$
\begin{gathered}
\|\delta \mathbf{E}(\mathbf{r})\| \leq \Upsilon, \\
\Upsilon:=M C \int_{S}\left\|\int_{U} \sum_{n, m}\right\| \delta \mathbf{F}_{n}^{1}\left(\mathbf{r}^{\prime \prime}, \mathbf{r}^{\prime}\right)\|\| \delta \mathbf{F}_{m}^{2}\left(\mathbf{r}^{\prime \prime}, \mathbf{r}^{\prime}\right)\left\|d s^{\prime}\right\| d s^{\prime \prime} .
\end{gathered}
$$

The number $Y$ is the required upper bound on the near field error caused by mutual coupling and its formula (16) is the main result of the present paper.

Some observations on the derived bound are in order. First, the integral $\int_{S} d s^{\prime \prime}$ is on the total surface area of the antenna system. Second, as can be seen from (11), the number $M$ does not depend on the antenna type, but is a property of the free space Green's function. Therefore, according to (11), $M$ can always be determined for any compact region $D \times S \subset V^{\text {exter }} \times S$. In other words, for antenna design, $M$ may be taken as fixed because the free space Green's function, in contrast to the antenna current Green's function, cannot be changed. Third, the last integral in (16) can be computed from the mutual coupling ACGF function obtained by full-wave analysis or measurement. This bound is then valid for any input field satisfying (7) and was obtained for the most general possible conditions of operation. We conclude then that to reduce near-field deviations due to coupling effects, the antenna engineer can either reduce 1) the size of the antenna

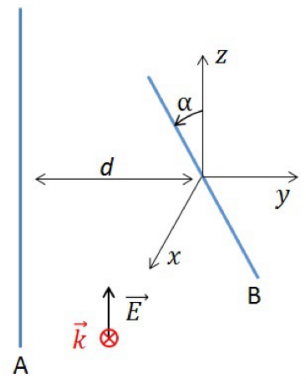

Fig. 1. The two thin wire antennas $\mathrm{A}$ and $\mathrm{B}$ with separation $d$. The wire $\mathrm{B}$ is rotated by $\alpha$ degrees in the xz-plane.

and/or 2) reduce the integral of the norm of the mutual coupling $A C G F$. In the following section we provide examples illustrating how the second option can be utilized in practice.

\section{NUMERICAL EXAMPLES}

For simplicity, we work with thin-wire antennas, where in Fig. 1, we show two linear wire antennas with separation $d$. One antenna will be rotated by angle $\alpha$ in order to control the shape of the mutual coupling ACGF. To obtain the ACGF, the energized antenna (A) is excited by a Dirac delta source at the middle of the wire. For wires, the ACGF itself has the simple functional form $\hat{z} \hat{z} F\left(z, z^{\prime}\right)$. When the antenna is rotated, we replace $\hat{z}$ by $\hat{u}$, where $\hat{u}$ is the unit vector along the antenna. The current $I(z)$ flowing along the wire can be written as $\mathbf{I}(z)=\hat{z} \int_{S} F\left(z, z^{\prime}\right) \hat{z} \cdot \mathbf{E}^{\mathrm{ex}}\left(z^{\prime}\right) d z^{\prime}$. Since the problem is onedimensional, a great simplification in the upper bound (16) can be obtained in this case. Indeed, for linear wires, the upper bound (16) reduces to the following

$$
\Upsilon=L M C \int_{S}|\delta F(z, 0)| d z
$$

where $L$ is the total length of the antenna. In terms of the notation in (16), the port is located at $z^{\prime}=0$. Comparing (17) with (16), we realize that the inner $s^{\prime}$-integral in (16) collapsed to mere evaluation of the ACGF at $z^{\prime}=0$ since a Dirac delta source is applied there. The integral in (17) is the area under the magnitude of the mutual coupling ACGF.

We consider two half-wavelength linear wire antennas at 10 GHz. A Method of Moment (MoM) code (checked by WIPLD) is used to approximate the ACGF of the system in the transmitting mode. In order to verify the mutual coupling ACGF, the antennas system (for $d=0.5 \lambda$ ) is then excited by a plane wave and a scattering MoM code is used to predict the induced current at the receiving port. The inverse reciprocity theorem [2] and (1) allow us to predict the same signal using the ACGF of the TX mode. The current received by the wire $\mathrm{A}$ as a function of the rotation of the wire $\mathrm{B}$ is presented in Fig. 2 computed both using the MoM and the ACGF formalism. The two results are in excellent agreement and the same conclusion applies for all separations $d$, including those resulting in strong mutual coupling. 


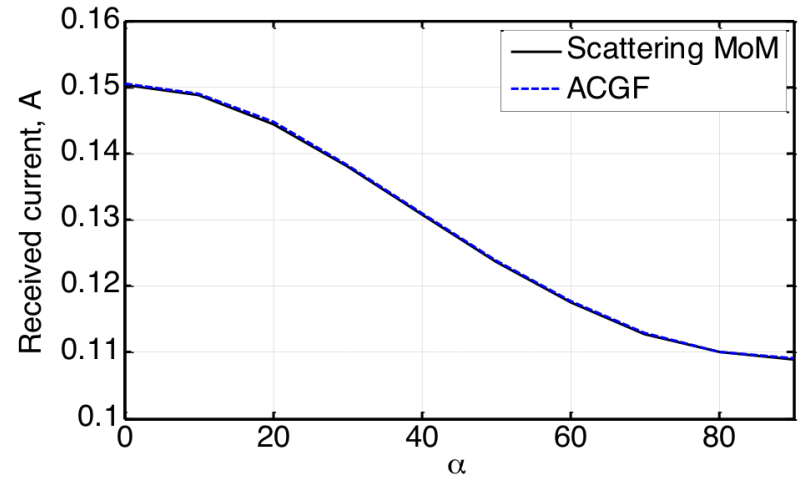

Fig. 2. Magnitude of the current received by the wire A when exciting by a plane wave as a function of the rotation of the wire $\mathrm{B}$, computing both using the MoM and ACGF formalism.

Moreover, the received current can be further split into three different parts: one due to the wire A without mutual coupling or $F_{0}^{A}\left(z, z^{\prime}\right)$; the second due to the wire A with mutual coupling effect or $\delta F^{A}\left(z, z^{\prime}\right)$; and the third due to the wire B or $F^{B A}\left(z, z^{\prime}\right)$. The contributions of these three ACGFs are presented in Fig. 3 and they illustrate the validity of (2) and (3). The current due to $F_{0}^{A}\left(z, z^{\prime}\right)$ is obviously constant because it does nott depend on the wire B. For the two other parts, their contributions decrease as the rotation $\alpha$ increases. Taking the size $L$ of the antennas as constant, we also fix $M$ and $C$ by normalization of the results (we investigate the evaluation of in future papers.)

For the purpose of the present letter, we focus mainly on the effect of the shape and topology of the array on NF mu- tual coupling. From (17), the normalized upper bound of the nearfield deviation caused by mutual coupling is computed for this system as a function of rotation and the results are presented in Fig. 4. It is seen here that, according to the derivations of (16), deviations in the near field for fixed antenna size can be reduced by reducing the area under the magnitude of mutual coupling ACGF. In this particular example, such reduction was attained by changing the shape of this ACGF, which in turn was achieved by changing the relative orientation of one antenna. More extensive approach will require using optimization methods where the norm in (16) stands for the

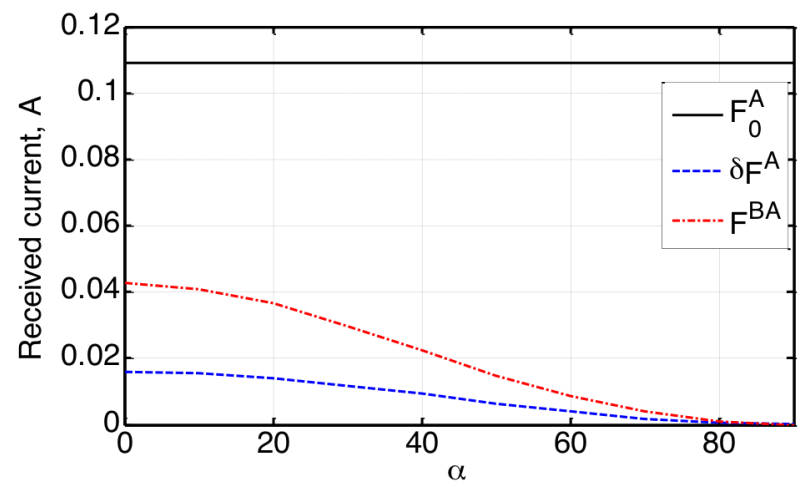

Fig. 3. Contribution of each part of the system into the current received by the wire as a function of the rotation of the wire B.

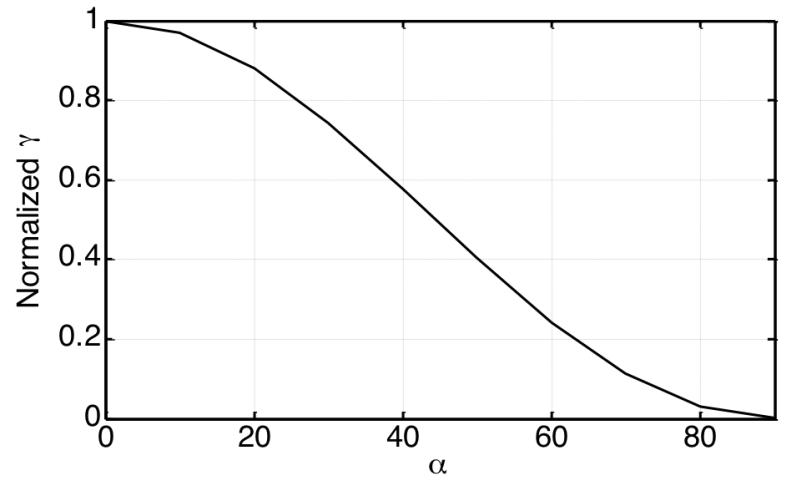

Fig. 4. Upper bound on the near-field error caused by mutual coupling as a function of the rotation of the wire B.

cost function to be minimized.

\section{CONCLUSION}

For a given antenna system size, an exact upper bound on near-field deviations was found to depend on the norm of the mutual coupling ACGF, the latter a function of the current on the entire antenna surface. The derived upper bound demonstrates that optimization methods aiming at reduction of near-field performance degradation can be achieved by working with the mutual coupling ACGF instead of the more complex near field it- self in the near zone. This letter provided a theoretical frame- work for further study of the physics of near-field devices from the perspective of the current on the entire surface of the radiating system.

\section{REFERENCES}

[1] T. B. Hansen and A. D. Yaghjian, Plane-Wave Theory of Time-Domain Fields: Near-Field Scanning Applications. New York, NY, USA: WileyIEEE Press, 1999

[2] S.M. Mikki and Y.M.M. Antar, "The antenna current green's function formalism—Part I, II," IEEE Trans. Antennas Propag., vol. 61, no. 9, pp. 4493-4519, Sep. 2013.

[3] S.M. Mikki and Y.M.M. Antar, "A rigorous approach to mutual coupling in general antenna systems through perturbation theory," IEEE Antennas Wireless Propag. Lett., vol. 14, pp. 115-118, 2015.

[4] S. Henault, S.K. Podilchak, S.M. Mikki, and Y.M.M. Antar,“ A general methodology for mutual coupling estimation and compensation," IEEE Trans. Antennas Propag., vol. 16, no. 3, pp. 1119-1131, Mar. 2013.

[5] S.M. Mikki and Y.M.M. Antar, "A theory of antenna electromagnetic near fields-Parts I, II,” IEEE Trans. Antennas Propag., vol. 59, no. 12, pp. 4691-4724, Dec. 2011.

[6] S. M. Mikki and Y. M. M. Antar, "Near-field analysis of electromagnetic interactions in antenna arrays using equivalent dipole models," IEEE Trans. Antennas Propag., vol. 60, no. 3, pp. 1381-1389, Mar. 2012.

[7] S. Clauzier, S. M. Mikki, and Y. M. M. Antar, "Design of near-field synthesis arrays through global optimization," IEEE Trans. Antennas Propag., vol. 63, no. 1, pp. 151-165, Jan. 2015.

[8] C. Balanis, Antenna Theory. Hoboken, NJ, USA: Wiley-Interscience, 2012.

[9] C. Craeye and D. González-Ovejero, "A review on array mutual coupling analysis," Radio Sci., vol. 46, no. 2, 2012, doi:10.1029/ 2010RS004518.

[10] A. D. Yaghjian, "Efficient computation of antenna coupling and fields within the near-field region," IEEE Trans. Antennas Propag., vol. 30, no. 1, pp. 113-128, Jan. 1982.

[11] A. K. Skrivervik and J. R. Mosig, "Analysis of finite phase arrays of microstrip patches," IEEE Trans. Antennas Propag., vol. 41, no. 8, pp. 1105-1114, Aug. 1993. 